\title{
Impact of Anastomotic Leakage on Overall and Disease-free Survival After Surgery for Gastric Carcinoma: A Systematic Review
}

\author{
PAOLO AURELLO ${ }^{1}$, MATTEO CINQUEPALMI ${ }^{1}$, NICCOLÒ PETRUCCIANI ${ }^{1}$, GIOVANNI MOSCHETTA ${ }^{2}$, \\ LAURA ANTOLINO $^{1}$, FEDERICA FELLI ${ }^{1}$, DIEGO GIULITTI ${ }^{1}$, GIUSEPPE NIGRI ${ }^{1}$, \\ FRANCESCO D'ANGELO ${ }^{1}$, STEFANO VALABREGA ${ }^{1}$ and GIOVANNI RAMACCIATO ${ }^{1}$ \\ ${ }^{1}$ General Surgery Unit, Department of Medical and Surgical Sciences and Translational Medicine, \\ St. Andrea University Hospital, Sapienza University of Rome, Rome, Italy; \\ ${ }^{2}$ Department of General Surgery, Fondazione Policlinico Universitario \\ Agostino Gemelli IRCCS, Catholic University, Rome, Italy
}

\begin{abstract}
Background/Aim: Gastric cancer is the fifth most frequently diagnosed cancer and the second most common cause of cancer-related death. The only potentially curative treatment is surgical resection, which is associated with potentially severe complications, such as anastomotic leakage. The aim of this systematic review was to evaluate the relationship between anastomotic leakage and overall and disease-free survival after surgery for gastric cancer. Materials and Methods: A systematic literature search was performed and 7 articles published between 2010 and 2019 were included, including a total of 7,167 patients. Results: Among the included studies the frequency of anastomotic leakage ranged from 6 to $41 \%$. Patients affected by anastomotic leakage had an overall survival ranging between 4.1 and 97.6 months, whereas patients who did not experience anastomotic leakage had an overall survival between 23 and 109.5 months. Conclusion: Closer follow-up or even more aggressive oncological therapy may be considered for patients affected by anastomotic leakage after surgery for gastric cancer.
\end{abstract}

Gastric cancer is the fifth most frequently diagnosed cancer and the second most common cause of cancer-related death

This article is freely accessible online.

Correspondence to: Dr. Cinquepalmi Matteo, General Surgery Unit, Department of Medical and Surgical Sciences and Translational Medicine, St. Andrea University Hospital, Sapienza University of Rome, Via di Grottarossa 1035, Rome, Italy. Tel: +39 0633776746, Fax: +39 0633775649, e-mail: matteo.cinquepalmi@uniroma1.it

Key Words: Gastric cancer, disease-free survival, overall survival, anastomotic leakage, review.
(1-2). In the world, annually, more than one million diagnoses of gastric cancer are made and the incidence of gastric cancer is highly dependent on region and culture (2). The cumulative risk of developing gastric cancer from birth to age 74 is higher in males, amounting for $1.87 \%$ against a cumulative risk of $0.79 \%$ in females worldwide.

The management of patients with gastric cancer is interdisciplinary, including surgical oncology, medical oncology, gastroenterology, radiation oncology, radiology, and pathology. Nowadays, surgery remains the primary treatment option for patients with resectable tumors $(2,3)$.

For patients with cT2 or even more advanced gastric tumors, perioperative chemotherapy and preoperative chemoradiation (category 2B) should always be considered, even if surgical resection with negative margins (R0) remains the most important step of the treatment (3).

However, gastric cancer surgery may be associated with several complications. The overall complication rate after radical gastrectomy for gastric cancer is $18.7 \%$. The most common complication is anastomotic leakage (AL), which affects up to $3.4 \%$ of patients $(3,4)$.

The long-term consequences of AL following gastric cancer surgery are still debating. The aim of our work was to analyse the impact of AL on disease-free-survival (DFS) and overall survival (OS) in patients who underwent gastrectomy for cancer, through a careful analysis of the results currently available in the literature.

\section{Patients and Methods}

Search strategies. We conducted a systematic search in PubMed, Embase, Cochrane Library, CILEA Archive, BMJ Clinical Evidence and Up ToDate databases with the following search terms: "cancer" AND "gastric" (or "stomach") AND "anastomotic leakage" (or 
Table I. Characteristics of the included studies with quality assessment (NOS score).

\begin{tabular}{|c|c|c|c|c|c|c|c|c|c|}
\hline First author year & $\begin{array}{c}\text { Study } \\
\text { type }\end{array}$ & Country & Histology & $\begin{array}{l}\text { Study } \\
\text { period }\end{array}$ & Patients, $\mathrm{n}$ & $\begin{array}{l}\mathrm{AL} \\
(\%)\end{array}$ & $\begin{array}{c}\text { Age } \\
\text { Years } \\
\text { median }\end{array}$ & $\begin{array}{c}\text { Gender } \\
\mathrm{M} / \mathrm{F}\end{array}$ & $\begin{array}{l}\text { NOS } \\
\text { score }\end{array}$ \\
\hline Tsusjimoto et al. (10) 2008 & RCS & Japan & $\mathrm{AC}$ & 1986-2005 & 1332 & 48 & 61.2 & $905 / 427$ & 7 \\
\hline Sierzega et al. (11) 2010 & RCS & Poland & $\mathrm{AC}$ & 1999-2004 & 690 & 41 & 63.7 & $458 / 232$ & 7 \\
\hline Han Mo Yoo et al. (12) 2011 & RCS & Korea & $\mathrm{AC}$ & $2000-2005$ & 478 & $32(6.7 \%)$ & 58 & $326 / 152$ & 9 \\
\hline Kim et al. (13) 2015 & RCS & Korea & $\mathrm{AC}$ & 2003-2012 & 3.827 & $72(1.88 \%)$ & & $2,602 / 1,225$ & 8 \\
\hline Samples et al. (14) 2015 & RCS & USA & $\mathrm{AC}$ & 2004-2012 & 102 & 6 & 62.9 & $74 / 28$ & 8 \\
\hline Andreou et al. (15) 2016 & RCS & Germany & $\mathrm{AC} \mathrm{S}$ & 2005-2012 & 471 & 41 & 65 & $353 / 118$ & 9 \\
\hline Barchi et al. (16) 2019 & RCS & Brazil & $\mathrm{AC}$ & 2009-2017 & 258 & $15(5.8 \%)$ & 62.3 & $180 / 78$ & 9 \\
\hline
\end{tabular}

RCS: Retrospective cohort study; AC: adenocarcinoma; S: squamous; AL: anastomotic leakage.

fistula or dehiscence) AND "overall survival " AND "disease-free survival " AND "recurrence" AND "long-term-results".

We also scanned all the reference lists to identify other relevant studies. Unpublished reports were not considered eligible.

Compliance with ethical standards. All procedures performed in the studies involving human participants were in accordance with the ethical standards of the institutional and/or national research committee and with the 1964 Helsinki declaration and its later amendments or comparable ethical standards.

Study selection. Inclusion criteria were: 1) articles written in English and published between January 1998 and June 2019. 2) Open or minimally invasive surgery with curative intent (with histological confirmation of R0 status). 3) Cases of gastric adenocarcinoma and/or gastroesophageal adenocarcinoma type III according to Siewert classification (5). 4) Studies mentioning overall survival and/or disease-free survival.

Exclusion criteria were: 1) treatment for disease recurrence; 2) case report; 3) letter to the Editor; 4) Review.

Data extraction and quality assessment. Two authors (GM, MC) independently screened the articles by title, abstract and keywords, and then selected and analysed the relevant articles. Any disagreement was resolved by discussion with the senior author (PA).

PRISMA statement guidelines for conducting and reporting systematic reviews were followed (6).

The research protocol was registered at the International Prospective Register of Systematic Reviews with the following registration number: CRD42019120781 (6). The Newcastle-Ottawa Scale (NOS) was used for quality assessment (7). Cochrane Collaboration guidelines were followed (8). Three broad perspectives were used to judge a study evaluating the selection of the study groups, comparability, ascertainment of either the exposure or outcome of interest for casecontrol or cohort studies, respectively $(7,8)$. Results of quality assessment scale (7) are reported in Table I. The study was designed and reported according to the STROCSS criteria (9).

\section{Results}

The literature search yielded 135,194 items; 135,001 were not considered suitable for abstract or integral reading, including duplicates removal. Then, 193 titles and abstracts were reviewed (Figure 1). A total of 180 papers were excluded because they did not match our inclusion criteria. Finally, only 13 articles were selected for full-text review and of these, six more were excluded for the following reasons: Five because they did not report OS or DFS rates in patients with occurrence of anastomotic leakage and one because it did not report AL rates. There was no disagreement regarding eligibility of full-text articles.

A total of seven articles fulfilled the selection criteria and were included in this systematic review (10-16). This pool of articles consisted of seven retrospective reports. Characteristics of the included studies are presented in Table I. Table II shows the outcomes.

A total of 7,167 adult patients who underwent surgery for gastric and GEJ cancer (type III according to Siewert Classification) were included.

Tsujimoto et al. (10) have evaluated the influence of postoperative infection on long-term survival after potentially curative gastrectomy in 1,332 patients. Two groups of patients were considered: 141 patients composed the first group, who had postoperative complications (10.6\%). The second group, without postoperative complications, included the other 1,191 patients. The OS of patients with stage II gastric cancer was $65.6 \%$ (no postoperative complications) versus $49.7 \%$ if they experienced postoperative complications $(p=0.04)$. OS was $41.2 \%$ for patients with stage III gastric cancer but if they experienced postoperative complications it dropped to $20.6 \%$, $(p=0.002)$. Multivariate analysis demonstrated that AL was significantly associated with cancer-specific survival (10).

Sierzega et al. (11) included 699 patients of whom 41 were affected by AL (5.8\%). The group with AL had 5-years DFS of 11 months. The group without complications had a 5 -year DFS of 19 months $(p=0.021)$. The OS was 4.1 months and 23 months, respectively ( $p=0.001)$.

Han Mo Yoo et al. (12) included 478 patients. AL was found in 35 patients $(6.7 \%)$. In the group of patients affected by leakage, the overall mean survival was significantly 


\begin{tabular}{|c|c|c|}
\hline $\begin{array}{l}\text { Item } \\
\text { no. }\end{array}$ & Item description & $\begin{array}{l}\text { Page } \\
\text { Number }\end{array}$ \\
\hline 1 & $\begin{array}{l}\text { Title: The words "cohort" and the area of focus should appear in the title } \\
\text { (e.g. disease, exposure/intervention or outcome). Whether the study is } \\
\text { retrospective or prospective should also be stated. }\end{array}$ & 1 \\
\hline $2 a$ & $\begin{array}{l}\text { Abstract - Introduction: The background and scientific rationale for the } \\
\text { research question. }\end{array}$ & $2-3$ \\
\hline $2 b$ & $\begin{array}{l}\text { Abstract - Methods: Describe the study design (cohort design, } \\
\text { retrospective or prospective, single or multi-centre, etc), what was done } \\
\text { to each group, how, when was it done and by whom. }\end{array}$ & $2-3$ \\
\hline $2 \mathrm{c}$ & $\begin{array}{l}\text { Abstract - Results: What was found. Give the results for the main } \\
\text { outcomes. }\end{array}$ & $2-3$ \\
\hline $2 d$ & $\begin{array}{l}\text { Abstract - Conclusion: What have we learned and what does it mean. } \\
\text { Where should future research go, }\end{array}$ & $2-3$ \\
\hline 3 & $\begin{array}{l}\text { Explain the scientific background and rationale for the cohort study. } \\
\text { What are objectives, research questions and the hypotheses. }\end{array}$ & $3-4$ \\
\hline $4 a$ & $\begin{array}{l}\text { Registration and ethics: State the research registry number in accordance } \\
\text { with the declaration of Helsinki - "Every research study involving } \\
\text { human subjects must be registered in a publicly accessible database } \\
\text { before recruitment of the first subject" (this can be obtained from; } \\
\text { ResearchRegistry.com or ClinicalTrials.gov or ISRCTN). Even } \\
\text { retrospective studies should be registered prior to submission. }\end{array}$ & - \\
\hline $4 b$ & $\begin{array}{l}\text { Ethical Approval: State whether ethical approval was needed and if so, } \\
\text { what the relevant judgement reference from the IRB or local ethics } \\
\text { committee was? If ethical approval was not needed, state why. }\end{array}$ & - \\
\hline $4 \mathrm{c}$ & $\begin{array}{l}\text { Protocol: Was a research protocol developed apriori? Where can it be } \\
\text { accessed. Was it published in a journal e.g. US Protocols, BMJ Open, } \\
\text { etc, if so, provide the reference. }\end{array}$ & - \\
\hline $5 \mathbf{a}$ & $\begin{array}{l}\text { Study design - State the research is a cohort study and whether } \\
\text { prospective or retrospective in design, whether single or multi-centre. }\end{array}$ & 4 \\
\hline $5 b$ & $\begin{array}{l}\text { Setting: Describe the setting(s)and nature of the institution in which the } \\
\text { patient was managed; academic, community or private practice setting? } \\
\text { Location(s), and relevant dates, including periods of recruitment, } \\
\text { exposure, follow-up, and data collection. }\end{array}$ & $4-5$ \\
\hline $5 c$ & $\begin{array}{l}\text { Cohort Groups: State the number of groups in the study. What } \\
\text { interventions will each group receive? }\end{array}$ & - \\
\hline 5d & $\begin{array}{l}\text { Sub-group-Analysis: Any planned sub-group analyses are specified / } \\
\text { Describe any methods used to examine subgroups and interactions. }\end{array}$ & - \\
\hline $6 \mathrm{a}$ & $\begin{array}{l}\text { Participants: State any eligibility (inclusion/exclusion) criteria and the } \\
\text { sources and methods of selection of participants. Describe length and } \\
\text { methods of follow-up. }\end{array}$ & $3-4$ \\
\hline $6 \mathrm{~b}$ & $\begin{array}{l}\text { Recruitment: State the methods of how patients or participants were } \\
\text { recruited to each group, over what time periods. }\end{array}$ & $3-4$ \\
\hline $6 \mathrm{c}$ & $\begin{array}{l}\text { Sample size calculation: Whether there was calculation of margin of } \\
\text { error or a prior analysis to determine study population, or mention of } \\
\text { how appropriate study sample was determined. }\end{array}$ & $3-4$ \\
\hline $7 \mathrm{a}$ & $\begin{array}{l}\text { Pre-intervention considerations - e.g. Patient optimisation: measures } \\
\text { taken prior to surgery or other intervention e.g. treating } \\
\text { hypothermia/hypovolaemia/hypotension in burns patients, ICU care for } \\
\text { sepsis, dealing with anticoagulation/other medications and so on. }\end{array}$ & - \\
\hline $7 b$ & $\begin{array}{l}\text { Types of intervention(s) deployed: To include reasoning behind } \\
\text { treatment offered (pharmacological, surgical, physiotherapy, } \\
\text { psychological, preventive) and concurrent treatments (antibiotics, } \\
\text { analgesia, anti-emetics, nil by mouth, VTE prophylaxis, etc). Medical } \\
\text { devices should have manufacturer and model specifically mentioned. }\end{array}$ & - \\
\hline $7 \mathrm{c}$ & $\begin{array}{l}\text { Peri-intervention considerations: Administration of intervention (what, } \\
\text { where, when and how was it done, including details for surgery; } \\
\text { anaesthesia, patient position, use of tourniquet and other relevant } \\
\text { equipment, preparation used, sutures, devices, surgical stage ( } 1 \text { or } 2 \\
\text { stage, etc) and operative time. Pharmacological therapies should include } \\
\text { formulation, dosage, strength, route and duration). Authors are } \\
\text { encouraged to use figures, diagrams, photos, video and other multimedia } \\
\text { to explain their intervention. }\end{array}$ & - \\
\hline $7 d$ & $\begin{array}{l}\text { Who performed the procedure(s): Operator experience for each group } \\
\text { (position on the learning curve for the technique if established, } \\
\text { specialisation and prior relevant training). }\end{array}$ & - \\
\hline
\end{tabular}

Figure 1. The STROCSS Guideline.

lower: 30.5 versus 96.2 months $(p<0.001)$. AL was an independent factor predictive for overall survival $(\mathrm{HR}=3.58$, 95\% CI=2.29-5.59).

Kim et al. (13) included a total of 3,827 patients. They reported an AL rate of $1.88 \%$. They did not report the OS, and they showed a median DFS of 97.6 months in patients 7e Quality control: What measures were taken to reduce inter or intraoperator variation. What measures were taken to ensure quality and consistency in the delivery of the intervention e.g. independent observers, lymph node counts, etc.

7f Post-intervention considerations - e.g. post-operative instructions and place of care: Important follow-up measures - diagnostic and other test results. Future surveillance requirements - e.g. imaging surveillance of endovascular aneurysm repair (EVAR) or clinical exam/ultrasound of regional lymph nodes for skin cancer.

8 Outcomes: What primary and secondary (if any) outcomes will be $3-4$ assessed and how are they defined. Definitions should be clear and precise. Appropriate references to validation of outcome measures used should be provided if they exist.

9 Statistical methods: Clearly outlined statistical tests used to compare the $3-4$ outcomes between an intervention group and a comparison group, state whether pre-existing differences and known confounders were controlled. The statistical package used should be mentioned.

10a Participants recruited with a flow diagram: Report numbers involved in 3-4 each group and use a flow diagram to show recruitment, nonparticipation, cross-over, withdrawal from the study with reasons.

10b Comparison between groups including a table: Provide a table comparing the demographic, clinical/prognostic features (comorbidities, tumour staging, smoking status, etc) and relevant socioeconomic characteristics of each group and whether numerical differences are significant (using p-values and/or confidence intervals as differences are significant (using p-values and/or confic
appropriate). Were the groups matched and if so, how.

10c Changes: Any changes in the interventions during the course of the study (how has it evolved, been altered or tinkered with, what learnin occurred, etc) together with rationale and a diagram if appropriate. Degree of novelty for a surgical technique/device should be mentioned and a comment on learning curves should be made for new techniques/devices.

11a Outcomes and follow-up: Clinician assessed and patient-reported outcomes (when appropriate) should be stated for each group (size of effect with raw numbers and percentages) with inclusion of the time periods at which assessed. Relevant photographs/radiological images should be provided e.g. 12 -month follow-up.Make it clear which confounders were adjusted for and which were not.

11b Intervention adherence/compliance and tolerability: How was this assessed. Describe loss to follow-up (express as a percentage and a fraction) or cross-over between group and any explanations for them.

11c Complications and adverse or unanticipated events: Describe in detail and ideally categorise in accordance with the Clavien-Dindo Classification. How they were prevented, mitigated, diagnosed and managed. Blood loss, wound complications, re-exploration/revision surgery, 30-day post-op and long-term morbidity/mortality may need to be specified.

12 Summatise key results.

13 Discussion of the relevance of the findings and rationale for conclusions: Relevant literature, implications for clinical practice conclusions: Relevant literature, implications for clinical practice
guidelines, how have the indications for a new technique/device bee refined and how do outcomes compare with established therapies and the prevailing gold standard should one exist and any relevant

hypothesis generation. The rationale for any conclusions.

14 Strengths and limitations of the study. 4-5

15 State what needs to be done next, further research with what study design(s).

16 State the key conclusions from the study and key directions for future 5 research

17a State any conflicts of interest. $\quad 5$

17b State any sources of funding

with AL and a median DFS of 109.5 months in patients, who did not experience AL $(p=0.076)$.

Samples et al. (14), collected data of 102 patients. Six ALs occurred (5.8\%). The 5-year DFS was not reported.

Andreou et al. (15) collected data from 471 patients. 41 patients were affected by AL (8.7\%), with a 5-year overall 
Table II. Primary outcomes of each included study.

\begin{tabular}{|c|c|c|c|c|c|c|}
\hline \multirow[t]{2}{*}{ Authors, year (Ref) } & \multirow{2}{*}{$\begin{array}{l}\text { Follow-up duration, } \\
\text { months (range) }\end{array}$} & \multirow[t]{2}{*}{$\mathrm{AL}, \mathrm{n}$} & \multicolumn{2}{|c|}{ 5-Year DFS $(\%)$} & \multicolumn{2}{|c|}{ OS (mo) } \\
\hline & & & $\mathrm{AL}$ & NL & $\mathrm{AL}$ & NL \\
\hline Tsusjimoto et al. (10) 2008 & $68.5(3-211)$ & $48(3.6 \%)$ & NR & NR & $\mathrm{NR} * 0.0015$ & NR \\
\hline Sierzega et al. (11) 2010 & $34(5-501)$ & $41(5.9 \%)$ & $\begin{array}{l}11 \mathrm{mo} \\
* 0.021\end{array}$ & 19 mo & $4.1 \mathrm{mo} * 0.001$ & $23 \mathrm{mo}$ \\
\hline Yoo et al. (12) 2011 & $62.5 \mathrm{mo}$ & $32(6.7 \%)$ & $\begin{array}{c}28.4 \\
* 0.001\end{array}$ & $\begin{array}{c}97.2 \\
* 0.001\end{array}$ & $30.5 \mathrm{mo}$ & $96.2 \mathrm{mo}$ \\
\hline Kim et al. (13) 2015 & $58.5(0-136)$ & $72(1.88 \%)$ & $\begin{array}{c}97.6 \\
* 0.076\end{array}$ & 109.5 & NR & NR \\
\hline Samples et al. (14) 2015 & $20.3 \mathrm{mo}$ & $6(5.8 \%)$ & NR & NR & & $\begin{array}{c}\text { Median OS } 70.3 \\
* 0.17\end{array}$ \\
\hline Andreaou et al. (15) 2016 & $35(1-101)$ & $41(8.7 \%)$ & $\begin{array}{c}35 \% \\
* 0.005\end{array}$ & $58 \%$ & $\begin{array}{l}39 \%(\mathrm{n}=41) \\
\quad * 0.005\end{array}$ & $61 \%(\mathrm{n}=430)$ \\
\hline Barchi et al. (16) 2019 & $25.5(1-102)$ & $15(5.8 \%)$ & NR & NR & $\begin{array}{l}50 \mathrm{mo} \\
* 0.013\end{array}$ & $100 \mathrm{mo}$ \\
\hline
\end{tabular}

AL: Anastomotic leakage; DFS: disease-free survival; OS: overall survival; NL: non anastomotic leakage; NR: non reported; mo: months; ${ }^{*} p$-value reported in the reference articles; $n=$ number of patients.

survival rate of $35 \%$ versus not $\mathrm{AL}$ of $58 \%(p=0.005)$. OS in patients with $\mathrm{AL}$ was $39 \%$ versus $61 \%$ in those without $\operatorname{AL}(p=0.005)$.

Barchi et al. (16) enrolled 258 patients. They had 15 patients with AL (5.8\%). In this manuscript the 5-year DFS was not reported. The OS was 50 months in patients with AL versus 100 months in patients without AL.

\section{Discussion}

The aim of our work was to analyse the impact of AL on DFS and OS after gastric cancer surgery. The relationship between $\mathrm{AL}$ and long-term results after surgery for gastric cancer is still the subject of debate. Our group has recently published a review regarding the relationship between $\mathrm{AL}$ and oesophageal cancer, showing how, with a proper literature analysis, is it possible to highlight the relationship between $\mathrm{AL}$ and survival (17). A similar investigation was performed on colorectal surgery, concluding that $\mathrm{AL}$ after proctectomy for cancer is associated with worse long-term DFS and overall survival (18).

Beecher et al. (19) have demonstrated in their review how surgical trauma, inflammatory response, intraoperative tumor manipulation, growth factors released during the procedure and tumor growth have a significant impact on OS and recurrence rates. Surgery can indeed bring to suppression cellmediated immunity and diffusion of malignant cells into the bloodstream, therefore, increasing the metastatic potential. In our research, five articles showed that AL had a statistically significant impact on the OS and DFS (10-12, 15, 16), and two articles written by Kim et al. and Samples et al. did not find any statistically significant relationship $(13,14)$.
The studies showing that AL had a statistically significant impact on OS and DFS included 3,238 patients, whereas the study that did not found any correlation between AL and long-term results $(13,14)$ included a total of 3,929 patients. In absolute numbers, articles showing no correlation between AL and worse long-term outcomes have a higher number of enrolled patients. However, the studies showing a significant correlation between AL and survival included more patients with AL: 273 patients had AL (8.4\%, range=5.8-10.6\%) in this group versus 78 patients $(1.9 \%$, range $=1.88-5.8 \%)$ in the articles not showing any significant relationship. The smaller number of patients with AL may limit the statistical power of studies not showing any correlation.

Indeed, a global analysis of the data shows that there is a negative trend in long-term outcomes (worse DFS and worse OS) in patients with AL.

Tsujimoto et al. (10), did not report OS as months of survival, but in the multivariate analysis they demonstrated the negative impact of AL on OS ( $p=0.0015)$. Moreover, they analysed the correlation between AL and cancer-specific survival finding a statistically significant correlation $(p=0.0358)$.

Sierzega et al. (11) demonstrated how AL has negative effects on OS and DFS. They showed that patients with AL had an OS of 4.1 months versus 23 months for patients who did not have AL $(p=0.001)$. Regarding DFS, they have shown that patients with AL had worse overall survival: 11 months compared to 19 months in patients without AL $(p=0.021)$. In this study, the factors predisposing to AL were also analysed; they demonstrated that poor performance status had the greatest impact as a predictive factor of AL.

Yoo et al. (12) demonstrated that AL has a negative impact on OS and DFS, in a multivariate analysis $(p<0.001)$. 
They also identified AL as one of the causes of cancerrelated death (32.2 vs. 99.5 months; $p<0.001)$, and recognized poor performance status as the most important factor predisposing to AL.

Andreau et al. (15) showed that AL is a significantly predictor of worse OS $(p=0.037)$ and DFS $(p=0.004)$. In their analysis, AL turned out to be an unfavourable factor independently from tumor stage and biology, and in the multivariate analysis for factors associated with tumor recurrence, AL was an independent predictor of tumor recurrence $(p=0.002)$.

Barchi et al. (16) demonstrated that OS is statistically worse in patients with AL than in non-AL patients. They reported 15 fistulas in 258 patients operated for gastric cancer, with decreased $\mathrm{OS}$ in patients with $\mathrm{AL}$ compared to non-AL (50 months and 100 months, respectively). Barchi et $a l$. recognized the type of surgery as a major cause of $\mathrm{AL}$ (total vs. completion gastrectomy).

Kim et al. (13) analysed a cohort of 3827 patients. They had 72 fistulas, $1.88 \%$ of the total number of included patients. They showed that patients with leakage and without leakage did not differ significantly in terms of DFS $(p=0.076)$. Their statistics also showed that the AL was not associated with cancer-related death.

Samples et al. (14), in their analysis, showed that there is no correlation between AL and worse OS.

During the selection of the articles, some were excluded because they did not strictly respect the inclusion criteria. They did not specifically investigate the relationship between AL and worse long-term survival, but they analysed the negative impact of $\mathrm{AL}$ on general complications, pathological stage, surgical technique, inflammation, and the long-term survival (20-27). The impact of $\mathrm{AL}$ on the prognosis of several cancers is still debated (28-31).

All the included studies were retrospective, carrying an intrinsic risk of selection bias, including heterogeneity linked to the changes in clinical practice, technology, surgical and chemoradiotherapy protocols. Indeed, changes in adjuvant chemotherapy practice may have included some selection bias, with patients treated in the most recent years showing better oncological outcomes due to more effective protocols. The definition of AL, reported in 5 out of 7 articles (11-16), appeared to be different among the studies, because it was based on different clinical parameters. In all the articles, AL, when recognized, was investigated radiologically. The different classification used for the gravity of AL evaluation could be a potential source of bias. Only 2 articles reported the Clavien-Dindo classification: Barchi et al. (16) reported $3 \mathrm{AL}$ of grade I-II and $12 \mathrm{AL}$ of grade III-V; Samples et al. (14) reported 6 ALs of grade III. Moreover, none of the articles stratified the risk based on tumor staging except for Barchi et al. (16).

\section{Conclusion}

The majority of reviewed studies showed an association between AL and DFS and OS. Closer follow up or an even more aggressive oncologic therapy may be considered for patients affected by postoperative anastomotic leakage.

\section{Conflicts of Interest}

None of the Authors had any conflicts of interest to declare.

\section{Authors' Contributions}

Paolo Aurello gave substantial contribution in the design of the work, drafting the work, final approval of the version to be published. Matteo Cinquepalmi, Niccolò Petrucciani and Giovanni Moschetta gave substantial contribution in the acquisition, analysis and interpretation of data for the work, drafting the work and final approval of the version to be published. Laura Antolino, Federica Felli, Diego Giulitti, Giuseppe Nigri, Francesco D’Angelo, Stefano Valabrega and Giovanni Ramacciato gave substantial contribution in critically revising the article for important intellectual content and final approval of the version to be published. All Authors agreed to be accountable for all aspects of the work in ensuring that questions related to the accuracy or integrity of any part of the work are appropriately investigated and resolved.

\section{References}

1 Jiang N, Deng JY, Ding XW, Zhang L, Liu HG, Liang YX and Liang H: Effect of complication grade on survival following curative gastrectomy for carcinoma. World J Gastroenterol 20(25): 8244-8252, 2014. PMID: 25009399. DOI: 10.3748/wjg.v20.i25.8244

2 Rawla P and Barsouk A: Epidemiology of gastric cancer: global trends, risk factors and prevention. Prz Gastroenterol 14(1): 2638, 2019. PMID: 30944675. DOI: 10.5114/pg.2018.80001

3 National Comprehensive Cancer Network (NCCN): Gastric Cancer. (Version 1.2019 - March 14, 2019). Available at: https://www.nccn.org/professionals/physician_gls/pdf/gastric.pdf

4 Tsou CC, Lo SS, Fang WL, Wu CW, Chen JH, Hsieh MC and Shen KH: Risk factors and management of anastomotic leakage after radical gastrectomy for gastric cancer. Hepatogastroenterology 58(105): 218-223, 2011. PMID: 21510318.

5 Kumamoto T, Kurahashi Y, Niwa H, Nakanishi Y, Okumura K, Ozawa R, Ishida $\mathrm{Y}$ and Shinohara H: True esophagogastric junction adenocarcinoma: background of its definition and current surgical trends. Surg Today, 2019. PMID: 31278583. DOI: $10.1007 / \mathrm{s} 00595-019-01843-4$

6 Chien PF, Khan KS and Siassakos D: Registration of systematic reviews: PROSPERO. BJOG 119(8): 903-905, 2012. PMID: 22703418. DOI: $10.1111 / \mathrm{j} .1471-0528.2011 .03242 . x$

7 Wells G, Shea B, O'Connel D, Peterson J, Welch V, Losos M and Tugwell P: The Newcastle-Ottawa Scale (NOS) for assessing the quality of non-randomized studies in meta-analysis. Available at: http //www. ohri ca/programs/clinical_epidemiology/nosgen.pdf

8 Higgins JPT and Green S (eds.): Cochrane handbook for systematic reviews of interventions version 5.1.0., 2011. Available at: http://www.cochrane-handbook.org 
9 Agha RA, Borrelli MR, Vella-Baldacchino M, Thavayogan R and Orgill DP; STROCSS Group. The STROCSS statement: Strengthening the reporting of cohort studies in surgery. Int J Surg 46: 198-202, 2017. PMID: 28890409. DOI: 10.1016 j.ijsu.2017.08.586

10 Tsujimoto H, Ichikura T, Ono S, Sugasawa H, Hiraki S, Sakamoto N, Yaguchi Y, Yoshida K, Matsumoto Y and Hase K: Impact of postoperative infection on long-term survival after potentially curative resection for gastric cancer. Ann Surg Oncol 16(2): 311-318, 2009. PMID: 19037699. DOI: 10.1245/s10434008-0249-8

11 Sierzega M, Kolodziejczyk P and Kulig J; Polish Gastric Cancer Study Group: Impact of anastomotic leakage on long-term survival after total gastrectomy for carcinoma of the stomach. Br J Surg 97(7): 1035-1042, 2010. PMID: 20632269. DOI: 10.1002/bjs. 7038

12 Han Mo Yoo MD, Lee HH, Shim JH, Jeon HM, Park CH and Song KY: Negative impact of leakage on survival of patients undergoing curative resection for advanced gastric cancer. J Surg Oncol 104(7): 734-740, 2011. PMID: 21792945. DOI: 10.1002/jso.22045

$13 \mathrm{Kim}$ SH, Son SY, Park YS, Ahn SH, Park DJ and Kim HH: Risk factors for anastomotic leakage: A retrospective cohort study in a single gastric surgical unit. J Gastric Cancer 15(3): 167-175, 2015. PMID: 26468414. DOI: 10.5230/jgc.2015.15.3.167

14 Samples JE, Snavely AC and Meyers MO: Postoperative morbidity in curative resection of gastroesophageal carcinoma does not impact long-term survival. Am Surg 81(12): 1228-1231, 2015. PMID: 26736158.

15 Andreou A, Biebl M, Dadras M Struecker B, Sauer IM, ThussPatience PC, Chopra S, Fikatas P, Bahra M, Seehofer D, Pratschke J and Schmidt SC: Anastomotic leak predicts diminished long-term survival after resection for gastric and esophageal cancer. Surgery 160(1): 191-203, 2016. PMID: 27067160. DOI: $10.1016 /$ j.surg.2016.02.020

16 Barchi LC, Ramos MFKP, Pereira MA, Dias AR, Ribeiro-Júnior U, Zilberstein B and Cecconello I: Esophagojejunal anastomotic fistula: a major issue after radical total gastrectomy. Updates Surg 71(3): 429-438, 2019. PMID: 31161587. DOI: 10.1007/ s13304-019-00659-8

17 Aurello P, Berardi G, Moschetta G, Cinquepalmi M, Antolino L, Nigri G, D'Angelo $F$, Valabrega $S$ and Ramacciato $G$ : Recurrence following anastomotic leakage after surgery for carcinoma of the distal esophagus and gastroesophageal junction: A systematic review. Anticancer Res 39(4): 1651-1660, 2019. PMID: 30952703. DOI: 10.21873/anticanres.13270

18 Park JS, Huh JW, Park YA, Cho YB, Yun SH, Kim HC and Lee WY: Risk factors of anastomotic leakage and long-term survival after colorectal surgery. Medicine (Baltimore) 95(8): e2890, 2016. PMID: 26937928. DOI: 10.1097/MD.0000000000002890

19 Beecher SM, O'Leary DP, McLaughlin R, Sweeney KJ and Kerin MJ: Influence of complications following immediate breast reconstruction on breast cancer recurrence rates. Br J Surg 103(4): 391-398, 2016. PMID: 26891211. DOI: 10.1002/bjs.10068

20 Wong J: Esophageal resection for cancer in The University of Hong Kong, Queen Mary Hospital, Hong Kong, China (19822008). J Thorac Dis 10(Suppl 16): S1843-S1844, 2018. PMID: 30026969. DOI: $10.21037 /$ jtd.2018.01.84

21 Tokunaga M, Tanizawa Y, Bando E, Kawamura T and Terashima M: Poor survival rate in patients with postoperative intraabdominal infectious complications following curative gastrectomy for gastric cancer. Ann Surg Oncol 20(5): 1575-1583, 2013. PMID: 23076557. DOI: $10.1245 / \mathrm{s} 10434-012-2720-9$

$22 \mathrm{Li}$ QG, Li P, Tang D, Chen J and Wang DR: Impact of postoperative complications on long-term survival after radical resection for gastric cancer. World J Gastroenterol 19(25): 40604065, 2013. PMID: 23840153. DOI: 10.3748/wjg.v19.i25.4060

23 Kubota T, Hiki N, Sano T, Nomura S, Nunobe S, Kumagai K, Aikou S, Watanabe R, Kosuga T and Yamaguchi T: Prognostic significance of complications after curative surgery for gastric cancer. Ann Surg Oncol 21(3): 891-898, 2014. PMID: 24254205. DOI: $10.1245 / \mathrm{s} 10434-013-3384-9$

24 Jiang N, Deng JY, Ding XW, Zhang L, Liu HG, Liang YX and Liang $\mathrm{H}$ : Effect of complication grade on survival following curative gastrectomy for carcinoma. World J Gastroenterol 20(25): 8244-8252, 2014. PMID: 25009399. DOI: 10.3748/ wjg.v20.i25.8244

25 Okumura Y, Hiki N, Kumagai K, Ida S, Nunobe S, Ohashi M and Sano T: Postoperative prolonged inflammatory response as a poor prognostic factor after curative resection for gastric cancer. World J Surg 41(10): 2611-2618, 2017. PMID: 28451762. DOI: $10.1007 / \mathrm{s} 00268-017-4032-5$

26 Powell A, Coxon AH, Patel N, Chan D, Christian A and Lewis W: Prognostic significance of post-operative morbidity severity score after potentially curative D2 gastrectomy for carcinoma. J Gastrointest Surg 22(9): 1516-1527, 2018. PMID: 29766446. DOI: $10.1007 / \mathrm{s} 11605-018-3787-9$

27 Hayashi T, Yoshikawa T, Aoyama T, Hasegawa S, Yamada T, Tsuchida K, Fujikawa H, Sato T, Ogata T, Cho H, Oshima T, Rino Y and Masuda M: Impact of infectious complications on gastric cancer recurrence. Gastric Cancer 18(2): 368-374, 2015. PMID: 24634097. DOI: 10.1007/s10120-014-0361-3

28 Aoyama T, Kazama K, Atsumi Y, Tamagawa H, Tamagawa A, Komori K, Machida D, Maezawa Y, Kano K, Hara K, Murakawa M, Numata M, Oshima T, Yukawa N, Masuda M and Rino Y: Clinical influence of anastomotic leakage on esophageal cancer survival and recurrence. Anticancer Res 40(1): 443-449, 2020. PMID: 31892599. DOI: 10.21873/anticanres.13972.

29 Koscielny A, Ko A, Egger EK, Kuhn W, Kalff JC and KeyverPaik MD: Prevention of anastomotic leakage in ovarian cancer debulking surgery and its impact on overall survival. Anticancer Res 39(9): 5209-5218, 2019. PMID: 31519635. DOI: 10.21873/ anticanres. 13718

30 Takahashi H, Haraguchi N, Nishimura J, Hata T, Yamamoto H, Matsuda C, Mizushima T, Doki Y and Mori M: The severity of anastomotic leakage may negatively impact the long-term prognosis of colorectal cancer. Anticancer Res 38(1): 533-539, 2018. PMID: 29277820. DOI: 10.21873/anticanres.12255

31 Sohda M, Kumakura Y, Saito H, Kuriyama K, Yoshida T, Honjyo H, Hara K, Ozawa D, Suzuki S, Tanaka N, Sakai M, Miyazaki T, Fukuchi $\mathrm{M}$ and Kuwano $\mathrm{H}$ : Clinical significance of salvage esophagectomy for patients with esophageal cancer and factors of influencing long-term survival. Anticancer Res 37(9): 5045-5051, 2017. PMID: 28870932. DOI: 10.21873/anticanres.11920

Received January 9, 2020

Revised January 17, 2020

Accepted January 20, 2020 